\title{
Shinren: Non-monotonic Trust Management for Distributed Systems ${ }^{\star}$
}

\author{
Changyu Dong and Naranker Dulay \\ Department of Computing \\ Imperial College London \\ 180 Queen's Gate, London, SW7 2AZ, UK \\ \{changyu.dong, n.dulay\}@imperial.ac.uk
}

\begin{abstract}
The open and dynamic nature of modern distributed systems and pervasive environments presents significant challenges to security management. One solution may be trust management which utilises the notion of trust in order to specify and interpret security policies and make decisions on security-related actions. Most logic-based trust management systems assume monotonicity where additional information can only result in the increasing of trust. The monotonic assumption oversimplifies the real world by not considering negative information, thus it cannot handle many real world scenarios. In this paper we present Shinren 1 , a novel non-monotonic trust management system based on bilattice theory and the any-world assumption. Shinren takes into account negative information and supports reasoning with incomplete information, uncertainty and inconsistency. Information from multiple sources such as credentials, recommendations, reputation and local knowledge can be used and combined in order to establish trust. Shinren also supports prioritisation which is important in decision making and resolving modality conflicts that are caused by non-monotonicity.
\end{abstract}

\section{Introduction}

The advances in communications and computing research have brought distributed systems and pervasive environments to new prominence. Applications are now distributed across the boundaries of networks, organisations, even countries and deployed on smaller mobile devices. The increasing scope of distributed applications also implies that applications must deal with "strangers" from other organisations and places. This leads to new challenges. How does the security system determine whether or not a request should be allowed if the request comes from an unknown user? The system must be able to decide without pre-knowledge of the user in order to authorise/deny the access. In other words, the system must determine whether and by how much does it trust a user. Trust management [1] was introduced in response to the challenges posed by modern distributed systems and pervasive environments.

In real-life, trust is normally non-monotonic. Consider the following:

\footnotetext{
* This research was supported by the UK's EPSRC research grant EP/C537181/1 (Caregrid) and EU FP7 research grant 213339 (ALLOW).

${ }^{1}$ Shinren: the pronunciation of trust in Chinese.
} 
"You are the CEO of a bank and looking for someone to manage a multi-billion pounds investment fund. A CV arrives on your computer. You quickly read through it: worked for the UK's oldest investment bank (interesting), had more than ten years experience as a derivatives trader (good), was the Chief Trader and General Manager of operations in futures markets on the Singapore Monetary Exchange (great), made $£ 10$ million a year which accounted for 10\% of former employer's annual income (excellent). You almost make up your mind. Then you see the candidate's name: Nick Leeson? Everything is turned upside down. You trash the email."

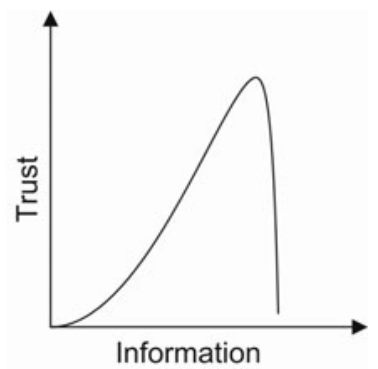

Fig. 1. Non-monotonic trust for CV

If we draw a diagram of this trust-information relation, it might look like Figure 1 From the diagram we can see that when new information comes in, trust can decrease, as well as increase, sometimes drastically. In other words, trust is non-monotonic. The non-monotonicity of trust is a natural consequence of the existence of both goodness and badness in the world. Trust, as defined by Mayer and Davis [2], is "the willingness of a party to be vulnerable to the actions of another party". To trust someone, the trustor needs to judge how competent, how honest, how dependable the trustee is, but more importantly, how incompetent, how dishonest and how undependable the trustee is. Positive information tells us how much we may gain from a trust relationship, while negative information tells us how much we may lose from it. Ignoring negative information may result in misplaced trust which may in turn cause serious damage to the trustor.

Although trust is non-monotonic, mainstream logic-based trust management systems [1 3 4 4 5 6 6] are monotonic. The reason is that complete knowledge is hard to achieve in large distributed systems and also that those systems are based on classical logic which cannot cope with this situation. Classical logic is monotonic which means a conclusion will never be retracted with new information, i.e. if $\Gamma \models \phi$ then $\Gamma \cup \gamma \models \phi$. To model trust with classical logic, a monotonic assumption is introduced to solve the problem and simplify the design by not using negative information about the world. Monotonic systems do not have problems with incomplete information because all missing information is positive and every decision that they make can only be more "correct" with more information. Accepting the monotonic assumption means accepting the world is always positive (however if there were no negative things in the world, do we still need

\footnotetext{
${ }^{2}$ Nicholas Leeson, the infamous rogue trader who caused the collapse of Barings Bank.
} 
trust management systems?). Monotonic trust management systems have many advantages, but the monotonic assumption is too limiting for many scenarios.

The gap between real world requirements and the current design of trust management systems motivates our work. A more realistic solution is needed for managing trust in distributed systems. In this paper, we describe Shinren, a novel non-monotonic trust management system based on bilattice theory and the any-world assumption. Shinren can make reasonable decisions even with incomplete information. Moreover, it can also utilise unreliable information which makes it more suitable for open distributed systems where reliable information is often hard to come by. Shinren does not just simply make use of the existing theories, it also supports prioritisation, which is achieved by a nontrivial extension of the original theories. Prioritisation is important in resolving conflicts and providing support for decision making.

This paper is organised as follows: We first summarise and compare existing nonmonotonic trust management systems with Shinren in section 2 . Then we discuss the motivation of this work in section 3 . In section 4 we introduce bilattice theory and the any-world assumption. In section 5, we describe Shinren, its policy language and present an example to show the details of policy evaluation in Shinren. In section 6 we show a prototype implementation. Section 7 concludes the paper.

\section{Non-monotonic Trust Management Systems}

Shinren is not the first logic-based non-monotonic trust management system. RuleControlled Environment For Evaluation of Rules and Everything Else (REFEREE ) [8], the Trust Establishment System (TES) from IBM [9] and $\mathrm{RT}_{\ominus}$ [10] are also nonmonotonic.

The main problem of existing logic-based non-monotonic trust management systems is semantics. A well-defined formal semantics is a critical part of any policy language. However, REFEREE and TES do not have formally defined semantics. $\mathrm{RT}_{\ominus}$ is based on the well-founded semantics which is a non-monotonic semantics proposed originally for logic programming with negation [11]. The problem with using well-founded semantics in trust management is that it is based on the closed world assumption (CWA) and the uniformity of CWA may lead to counter-intuitive results. For example, here is a simple trust policy $\operatorname{trust}(a):-\neg b a d(a)$. Under the well-founded semantics, when $\operatorname{bad}(a)$ is missing or not provable, it is falsified and thus makes trust( $a)$ true. However, this decision may seem too casual, especially when it is related to security. In Shinren, policy makers can use unknown as the default value for bad( $a$ ) while still use false as the default value for other positive atoms.

Existing non-monotonic trust management systems are also less expressive than Shinren. For example, $\mathrm{RT}_{\ominus}$ can only express policies using credentials. Among them, REFEREE is the most expressive one. It is capable of expressing policies utilising evidence from different sources, but it is incapable of distinguish decisions based on information of different quality. REFEREE is based on 3-valued logic, therefore there is no difference between a decision based on a statement from an authority and a decision based on rumour. The users of REFEREE may be given an unreliable trust decision 
without warning. In Shinren, a trust decision comes with a value which tells the user not only how true the decision is, but also how reliable it is.

Many systems attempt to assign real values to trust and develop sophisticated mathematical models to calculate trust values [12 13 14]. The values are usually based on past experience. Although they are also called trust management systems, we view them as a totally different approach from the trust management systems presented above which rely mostly on logical reasoning and view trust decisions as logical consequences of certain facts and theories. Quantitative trust management systems are usually nonmonotonic and can provide valuable information. However, the accuracy of the trust values largely depends on the amount of data input and may take a long time to get enough data. To differentiate, we call them reputation systems and Shinren can include such systems as subsystems.

\section{Why Non-monotonic? Why Shinren?}

So why do we need non-monotonic trust management systems? This is because (1) in the real world trust is non-monotonic and therefore a trust management system should be able to capture this; (2) monotonic assumption is not necessary in trust management, it is introduced merely because systems reasoning with classical logic cannot cope with the non-monotonicity in trust. The assumption does not solve the problem, it just makes systems ignore the problem. There are at least two bad consequences of monotonic assumption: first, a trust management system which can be proved correct under the monotonic assumption may not be correct in the real world because the assumption does not hold in general; second, it makes trust management systems incapable of handling certain real world scenarios.

Under the monotonic assumption, monotonic trust management systems do not consider negative information. Syntactically, this is achieved by not allowing negations in the policies. Negation-free policies work fine in some cases, however they reflect a limited view of the world and are inappropriate in many cases. For example, negationfree policies are quite inconvenient in handling exceptions. In the world modelled by negation-free policies, it is quite hard to express, "trust all the police officers except the bad ones" because without negations, we would be allowed to say "trust police officers" but not "do not trust the bad police officers". In the extreme case, we must specify for each individual good police officer a trust policy in order to exclude the bad ones. Lacking the ability for specifying exceptions can be dangerous particularly in trust management systems where delegations are used. No exceptions means that decisions have to be fully delegated to a delegatee, and the system must fully accept the delegatee's opinions. No exceptions also means that the system cannot accept part of the delegatee's decision while declining other parts. In other words, the system loses control after delegation. Another case is that negation-free policies cannot handle $m u$ tual exclusion. Coke is tasty, orange juice is tasty too. But the mixture of the two does not taste so pleasant. There are many examples that are mutually exclusive. However, with negation-free policies, there is no way to express " $\mathrm{A}$ is good, B is good, but $\mathrm{A}+\mathrm{B}$ is not good". In terms of security policies, separation of duties and conflict of interests are the most significant examples of this type of policy. 
One may argue that in the real world, people try to hide their negative aspects. Therefore, even if policies are allowed to use negative information, if the system cannot find it, the non-monotonic feature is useless. It is true that the information we can collect is always limited. But consider the following:

In monotonic trust management systems : trust :- good

$$
\begin{array}{r}
\text { In Shinren : distrust :-bad } \\
\text { trust :-good }
\end{array}
$$

What is the difference? When the system cannot find bad, i.e. the negative information, Shinren can behave exactly as the monotonic ones. However, because it is not possible to use negative information in monotonic trust management systems, their decisions will still be trust even if bad is presented! In contrast, Shinren's decision will no longer be trust because the distrust policy is applied. Although not guaranteed, Shinren aims to limit any damage with its best effort approach rather than silently ignoring it.

By using bilattices, Shinren suffers less from a dilemma which all trust management systems must face: on the one hand, in order to make a correct trust decision, a large amount of information is needed; on the other hand, in order to make the decision correct, most of the information available cannot be used because it is not reliable. Shinren can reason with unreliable information even with contradictory information. Monotonic trust management systems cannot. This ability is especially important in acquiring negative information.

Prioritisation is not present in any trust management systems. The philosophy is that sometimes trust is not just a Yes/No decision, but also a choice. You might want to follow one rule even if there are multiple rules you can follow, you might trust someone even if there are several persons you can trust. Prioritisation allows policy makers to specify their preferences and thus make complex policies possible. And also, in the presence of modality conflicts, prioritisation seems to be the only way to resolve them. Although there are overheads in defining and managing policies when using prioritisation, the overheads are minimised in Shiren because policy makers only assign priorities to local policies and only trust (distrust) policies are prioritised.

\section{Preliminaries}

\subsection{Bilattices}

Bilattice theory [15] was introduced by Ginsberg in the 1980s, and has been widely used in non-monotonic reasoning, knowledge representation and artificial intelligence. Bilattice is a non-empty, possibly infinite set of values with two partial orders, each one giving the set the structure of a lattice. A lattice $\langle L, \preceq\rangle$ is a non empty set $L$ along with a partial order $\preceq$ where any pair of elements $l_{1}, l_{2} \in L$ has a least upper bound (join) and a greatest lower bound (meet) in terms of $\preceq$. We write $l_{1} \prec l_{2}$ if $l_{1} \preceq l_{2}$ and $l_{1} \neq l_{2}$.

A bilattice, denoted by $\left\langle\mathcal{B}, \preceq_{t}, \preceq_{k}\right\rangle$ where $\mathcal{B}$ is a non-empty set and $\preceq_{t}, \preceq_{k}$ are two partial orders called the truth-order and the knowledge-order respectively. $\preceq_{t}$ is an ordering on the "degree of truth". $b_{1} \preceq_{t} b_{2}$ means $b_{2}$ represents at least as much truth as 
$b_{1}$ (and possibly more). Meet and join under $\preceq_{t}$ are denoted by $\wedge$ and $\vee$ and correspond to the classical conjunction and disjunction. $\preceq_{k}$ is an ordering on the "degree of knowledge". Meet and join under $\preceq_{k}$ are denoted by $\otimes$ and $\oplus$. $b_{1} \otimes b_{2}$ corresponds to the maximal information $b_{1}$ and $b_{2}$ can agree on, while $b_{1} \oplus b_{2}$ combines the information represented by $b_{1}$ and $b_{2}$.

The class of bilattice that we consider in this paper is restricted to interlaced bilattices. Interlaced bilattices are bilattices which satisfy the following: (1) if $b_{1} \preceq_{t} b_{2}$ then $b_{1} \otimes b_{3} \preceq_{t} b_{2} \otimes b_{3}$ and $b_{1} \oplus b_{3} \preceq_{t} b_{2} \oplus b_{3}$; (2) if $b_{1} \preceq_{k} b_{2}$ then $b_{1} \wedge b_{3} \preceq_{k} b_{2} \wedge b_{3}$ and $b_{1} \vee b_{3} \preceq_{k} b_{2} \vee b_{3}$. Thus in an interlaced bilattice an operation associated with one of the lattice orderings is required to be monotonic with respect to the other lattice ordering. This relates the two orderings. An alternative way of connecting the two orderings is via negation which reverses the truth ordering and is monotonic regarding the knowledge ordering.

Such bilattices can be constructed in a natural way by combining two lattices. Given two lattices $\left\langle L_{1}, \preceq_{1}\right\rangle$ and $\left\langle L_{2}, \preceq_{2}\right\rangle$, we can construct an interlaced bilattice as $\left\langle L_{1} \times\right.$ $\left.L_{2}, \preceq_{t}, \preceq_{k}\right\rangle$, where $\left(x_{1}, y_{1}\right) \preceq_{t}\left(x_{2}, y_{2}\right)$ if $x_{1} \preceq_{1} x_{2}$ and $y_{2} \preceq_{2} y_{1},\left(x_{1}, y_{1}\right) \preceq_{k}$ $\left(x_{2}, y_{2}\right)$ if $x_{1} \preceq_{1} x_{2}$ and $y_{1} \preceq_{2} y_{2}$. Negation can be defined as $\neg(x, y)=(y, x)$ if $L_{1}=L_{2}$. As we will see later, the bilattice used in our system is constructed in this way. We will expand on this later.

\subsection{Any-World Assumption}

Non-monotonic logics allow a conclusion to be drawn on incomplete information. One way of doing such reasoning is to complete the missing part by assumptions. Taking into account assumptions means assigning truth values, implicitly or explicitly, to the unknown facts. The assumptions are usually based on the estimated states of the facts. One of the most common assumptions is the Closed World Assumption (CWA). It assumes the default truth states of atoms to be false, therefore any atoms that cannot be proved to be true are taken as false. Another well-known assumption is the Open World Assumption (OWA). OWA is a more cautious assumption in the sense that it assumes the default truth states of atoms to be unknown. Therefore, any atoms that cannot be proved to be true are taken as unknown. However it also gives us less useful conclusions. Using only one of these assumptions to represent the world uniformly is usually not appropriate.

The Any-World Assumption (AWA) [16] unifies and extends the CWA and OWA by taking truth values from an arbitrary bilattice truth space and allow the default value of an atom to be any one of them. If in the assumptions, all the atoms are assigned to false, then it becomes CWA which says everything that cannot be inferred is false. If in the assumptions, all the atoms are assigned to unknown, then it becomes OWA which says everything cannot be inferred is unknown. The advantages are obvious: the truth, incompleteness and uncertainty can be represented in a finer granularity according to the experience and background information, therefore the assumptions we make carry more knowledge than before which in turn leads to more informed conclusions. The assumptions can be non-uniform which means the default truth values can vary for different atoms. This allows us to form more realistic assumptions. 


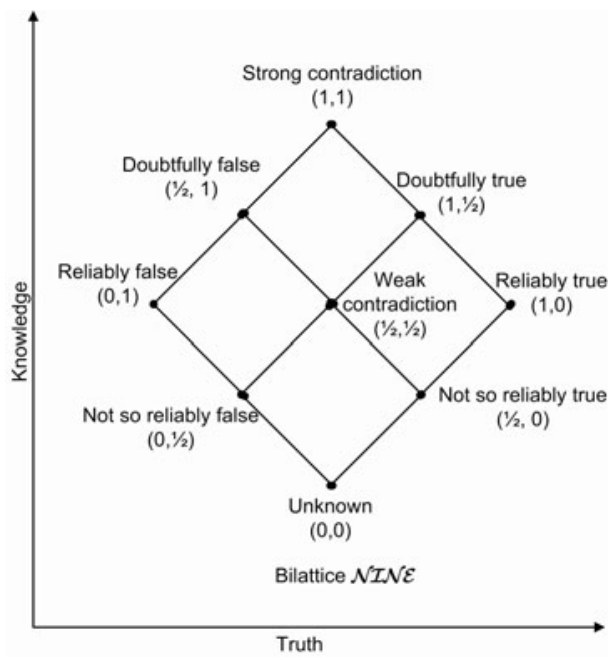

Fig. 2. The bilattice NINE

\section{Shinren}

\subsection{Bilattice $\mathcal{N} \mathcal{I} \mathcal{N E}$}

As introduced in Section 4.1, a standard way of constructing an interlaced bilattice is by combining two lattices. The bilattice we employ, $\mathcal{N} \mathcal{I N E}$, is also built in this standard way. $\mathcal{N} \mathcal{I N E}$ is obtained by combining two identical lattices $L_{1}=L_{2}=\left\langle\left\{0, \frac{1}{2}, 1\right\}, \leq\right\rangle$ where $\leq$ is "less than or equal". The structure of $\mathcal{N} \mathcal{I N E}$ is shown in Figure 2], The truth values are represented as tuples $(x, y)$ where $x, y \in\left\{0, \frac{1}{2}, 1\right\}$. The two orderings, $\preceq_{t}, \preceq_{k}$ are defined as:

$$
\begin{aligned}
& \left(x_{1}, y_{1}\right) \preceq_{t}\left(x_{2}, y_{2}\right) \text { if } x_{1} \leq x_{2} \text { and } y_{2} \leq y_{1} \\
& \left(x_{1}, y_{1}\right) \preceq_{k}\left(x_{2}, y_{2}\right) \text { if } x_{1} \leq x_{2} \text { and } y_{1} \leq y_{2}
\end{aligned}
$$

Given a statement with a truth value of the form $(x, y)$, the intuitive meaning of the truth value is that $x$ represents how much the statement is true (or you believe it is true), and $y$ represents how much the statement is false (or you believe it is false). For example, $(1,0)$, i.e. reliably true, is given to a statement supported by very strong and reliable evidence. The possibility of the statement is actually false can be neglected.

From the above it is easy to understand the two orderings. For example, a statement which is reliably true contains more truth (or is more likely to be true) than a statement which is not so reliably true, i.e. $\left(\frac{1}{2}, 0\right) \preceq_{t}(1,0)$. On the other hand, a reliably true statement gives us more information than a not so reliably true statement, i.e. $\left(\frac{1}{2}, 0\right) \preceq_{k}(1,0)$. It is possible to extend the bilattice to a finer model of reliability or uncertainty. For example, using a lattice with the value domain $\left\{0, \frac{1}{3}, \frac{2}{3}, 1\right\}$, we can create a bilattice with 16 truth values that can represent more reliability levels. However, we do not do so for two reasons: first, enlarging the bilattice also increases the 
computational complexity. With enough expressiveness, we would like to avoid unnecessary cost; second, things like reliability and uncertainty cannot typically be measured precisely. There are no metrics and instruments we can use to standardise the measurement. A finer scale does not help in solving this problem, even worse, it may bring a false sense of precision. For these reasons, we stay with this basic form and extend it when it is necessary and possible.

Let us also explain the rationale behind this multi-valued truth space. Classical logic, which is the basis of many trust management systems, is bivalent, i.e. the only possible truth values are true and false. It gives rise to "black and white thinking" where every proposition must be ascribed to "absolutely true" or "absolutely false". However, in the real world, many would agree with the statement "the only certainty is nothing is certain' 3 . Because classical logic lacks the ability of coping with the uncertainty in truth, mainstream trust management systems restrict the information that can be taken into account to "credentials". A credential is a statement signed by an issuer containing certain information about the credential holder and is believed to be highly reliable. The problems with credentials are two-fold: first, credentials are not able to carry every bit of information about the holder. We may find that signed information is just a very small fraction of all the information we can get. Second, in practice we do not encode negative information about the holder in credentials. The reason is simple: no one bothers to ask for a credential which is useless or has a negative effect to him. Again, we usually recognise a rogue merchant not from a "rogue merchant" credential signed by a government agency, but from various other sources like reviews in internet forums. If we want a more complete view of the trustee, using only credentials is not sufficient. We need to consider more information, possibly even that from the sources which are not so reliable. The multi-valued truth space gives Shinren the ability to represent and differentiate information with different qualities. And makes it possible for Shinren to utilise unreliable information.

The meet and join operators in terms of both orderings and the negation operator are then defined as follows:

$$
\begin{gathered}
\left(x_{1}, y_{1}\right) \vee\left(x_{2}, y_{2}\right)=\left(\max \left(x_{1}, x_{2}\right), \min \left(y_{1}, y_{2}\right)\right) \\
\left(x_{1}, y_{1}\right) \wedge\left(x_{2}, y_{2}\right)=\left(\min \left(x_{1}, x_{2}\right), \max \left(y_{1}, y_{2}\right)\right) \\
\left(x_{1}, y_{1}\right) \oplus\left(x_{2}, y_{2}\right)=\left(\max \left(x_{1}, x_{2}\right), \max \left(y_{1}, y_{2}\right)\right) \\
\left(x_{1}, y_{1}\right) \otimes\left(x_{2}, y_{2}\right)=\left(\min \left(x_{1}, x_{2}\right), \min \left(y_{1}, y_{2}\right)\right) \\
\neg(x, y)=(y, x)
\end{gathered}
$$

We will explain these with some examples. Given a statement $p$ which is reliably true and $q$ that is not so reliably true, the truth value of their conjunction is $p \wedge q=(1,0) \wedge$ $\left(\frac{1}{2}, 0\right)=\left(\min \left(1, \frac{1}{2}\right), \max (0,0)\right)=\left(\frac{1}{2}, 0\right)$, i.e. not so reliably true. This is easy to understand. Let $p$ be "Alice is a student" and $q$ be "Alice is a research assistant", then the statement "Alice is both a student and a research assistant" cannot be very reliable because we are not quite sure about the fact that she is a research assistant. Consider another example: in the court of a murder case, the prosecutor submits a CCTV record as evidence showing that the suspect was at the crime scene when the murder was happening, while the counsel of the suspect has a witness, who is a friend of the suspect,

\footnotetext{
${ }^{3}$ Pliny the Elder, Roman scholar (23-79 AD).
} 
to certify that the suspect was in a pub $50 \mathrm{~km}$ away from the scene at the same time. It turns out the conclusion of whether the suspect was at the scene after we combine these two pieces of evidence is: $(1,0) \oplus\left(0, \frac{1}{2}\right)=\left(\max (1,0), \max \left(0, \frac{1}{2}\right)=\left(1, \frac{1}{2}\right)\right.$. That is, although doubtful, we would believe the suspect is at the scene. The reason is that the video record is more reliable evidence.

\subsection{Shinren Policy Language}

The syntax of Shinren is based on the logic programming language Datalog [17], with certain extensions. As in Datalog, we do not have function symbols. The restriction is necessary to ensure finiteness of models and termination of inference. A rule, or policy, is of the form:

$$
A:-\varphi_{1}, \ldots, \varphi_{n} .
$$

where $A$ is an atom and each $\varphi_{i}$ is a literal, a consensus formula or a gullibility formula. ": -" is taken as " $\longleftarrow$ " and "," is taken as " $\wedge$ ". The atom $A$ on the left-hand side of the rule is called its head and the conjunction $\varphi_{1}, \ldots, \varphi_{n}$ on the right-hand side is called its body. Certain types of rules may also have a priority label $\langle l a b\rangle$ attached before the rules (will explain later). An assertion is a special type of rule defined as:

$$
A:-b \text {. }
$$

where $A$ is a ground atom and $b$ is a truth value. An assertion can be understood as $A$ has a truth value $b$. A fact set is a finite set of assertions. An assumption set is also a finite set of assertions. The difference is that the fact set contains the real truth values for the atoms while the assumption set contains the assumptions, i.e. assertions about the default values of the atoms. The assumptions are used only when no facts about the atoms can be found in the fact set or be inferred. We do not need to explicitly represent assumptions of the form $A:-(0,0)$. If no assumption about an atom can be found in the assumption set, the default value is $(0,0)$. A program is the union of a finite set of rules, a fact set and an assumption set.

By using the Shinren trust policy language, policy makers can define both trust policies and distrust policies, i.e. rules whose heads are trust or distrust predicates. They can also label the policies with priority levels. The priority levels express how preferable a policy is. The priority levels in Shinren language are defined as a finite set of nonnegative integers $\{0,1, \ldots, n\}$. 0 is reserved for default assignment rules. The higher the number is, the higher the priority is. For each priority level, policy makers also define two thresholds in terms of $\preceq_{t}$ or $\preceq_{k}$ or both, one for distrust policies and one for trust policies. The thresholds are used to filter poor answers. Answers that satisfy the threshold are called admissible answers. Note that only trust or distrust policies need labels, the other policies are not prioritised. When the system is asked to evaluate trust, it starts from policies with the highest priority. At the same level, distrust policies are evaluated before trust policies. In other words, distrust policies have a higher priority than trust policies at the same priority level. If an admissible answer can be found, then the evaluation ends. Otherwise it continues to evaluate the trust policies at the same level. If there are still no admissible answers, the system continues with the policies at the next level. When an admissible answer is found with truth value $b$, an answer for its counterpart is 
asserted with a truth value $\neg b$. For example, if the evaluation ends with an admissible answer $\operatorname{distrust}(\boldsymbol{a})=\left(\frac{1}{2}, 0\right)$, we also have $\operatorname{trust}(\boldsymbol{a})=\left(0, \frac{1}{2}\right)$. If after evaluating all the policies at higher priority levels, an admissible answer is still not found, the default value is applied. The default value assignment rules may be omitted, in this case the default value is $(0,0)$.

The prioritisation mechanism can be used to resolve modality conflicts introduced by trust and distrust policies. Trust and distrust are semantically opposite and it is possible in some situations that both are true based on the policies. Therefore we need to handle the possible conflicts. With priority levels, the conflicts can be resolved by "interlacing" distrust and trust policies and the decisions are governed by the policies with the highest priority levels which give admissible answers. The priority levels can also be used to order trust decisions. For example, if we have decided both Alice and Bob can be reliably trusted, we may prefer Alice if the decision about her came from a trust policy with a higher priority level, i.e. a more preferable policy. The truth values and priority levels can give hints to the decision maker. If the decision is not reliable or from a less preferable policy, it may indicate that the decision is not favourable and may be risky. The decision maker can activate some compensative controls based on the truth value and priority levels.

In order to achieve prioritisation, we require the program to be locally stratified, i.e. there are no cyclical dependencies between ground atoms. This syntactical restriction is needed to guarantee the policies can be evaluated correctly.

Due to space limit, the formal semantics of Shiren is omitted here but is available in the full version [18]. In next section, we will show the policy language by an example. Another example can be found in the appendix.

\subsection{Example 1: Electronic Marketplace}

Alice is a big fan of Internet shopping and she often visits a website called tBay which is an electronic marketplace like e-bay. Although she has bought a lot of items with very low prices, she also had several unpleasant experiences. So she wants to be cautious before she bids on anything from the website. She decides that she will only bid on items from sellers who live in the UK, have been registered no less than 6 months and have at least $80 \%$ positive feedback. She will also ask her friend Bob about his opinion and will not consider a seller if Bob does not like him. However, she knows tBay has a special procedure for items with bid prices lower than $£ 20$ : in case of dispute, tBay will fully refund the buyer. Since she is not going to lose money, Alice is willing to bid in such situations regardless of her other constraints above. But Alice also has a more important principle: she will never trade with someone who has cheated her. She has a blacklist of such sellers. Alice's policies are:

$\langle 3\rangle \operatorname{distrust}(X$, bid, Item $):-$ inBlackList $(X)$.

$\langle 2\rangle \operatorname{trust}(X$, bid, Item $):-\operatorname{soldBy}(X$, Item $)$, itemPrice(Item, Price), Price $\leq 20$.

$\langle 1\rangle \operatorname{distrust}(X$, bid, Item $):-\neg$ recommendation (bob, $X$, bid, Item).

$\langle 1\rangle \operatorname{trust}(X$, bid, Item) :- seller (tBay, X, Location, RegisterPeriod),

Location $=u k$, RegisterPeriod $\geq 6, \operatorname{soldB} y(X$, Item $)$,

reputation $(X$, goodSeller, tBay, $Y), Y \geq 0.8$. 
Alice's policies have 3 priority levels. At the highest level is the policy which should not be overridden by any other policies. At the second level is a trust policy that allows her to interact with any seller when there is no risk. The lowest level has two policies for general cases. In the policies, seller (tBay, X, Location, RegisterPeriod) represents a seller credential signed by tBay. $\operatorname{soldB} y(X$, Item $)$, itemPrice (Item, Price) and inBlackList $(X)$ are local knowledge predicates supplying useful information. For each priority level, Alice defines thresholds for admissible answers to be $(0,0) \prec_{t}$, which means only answers somehow true (reliably true, not so reliably true, doubtfully true) will be admissible.

Along with the policies, Alice also has a set of assumptions:

$$
\begin{aligned}
& \text { soldBy }(X, \text { Item }):-(0,1) . \\
& \text { itemPrice }(\text { Item, Price }):-(0,1) . \\
& \text { inBlackList }(X):-(0,1) .
\end{aligned}
$$

Recall that $(0,1)$ means "reliably false". Alice's assumptions are: if she cannot find any information that says an item is sold by seller $X$, then this item is not sold by $X$; if she cannot find any information that says an item is sold for a certain price, then it is not sold for this price; if she cannot find a seller in her blacklist, then he is not in her blacklist. These are easy to understand. All the other predicates are left with default values of $(0,0)$, i.e. unknown. Different default values may make a big difference. For example, if Alice assumes recommendation (bob, $X$, bid, Item) to be false, i.e. add recommendation (bob, $X$, bid, Item ) :- $(0,1)$ to her assumption set, then she cannot bid anything with a price higher than $£ 20$ when she cannot contact Bob. In such cases, since she cannot get recommendations from Bob, the default value will be used and the policy

distrust $(X$, bid, Item) :-ᄀrecommendation(bob, X, bid, Item).

will always give results of "distrust" with truth value $(1,0)$.

More complicated policies are also possible. For example, if Alice has another policy which says she will bid if at least two of her friends recommend the seller. This can be written as:

$$
\begin{aligned}
& \operatorname{trust}(X, \text { bid, Item }):-\operatorname{friend}(F 1), \text { friend }(F 2), \\
& \quad \text { recommendation }(F 1, X, \text { bid, Item }) \otimes \operatorname{recommendation~}(F 2, X, \text { bid, Item }) .
\end{aligned}
$$

Alice collects the following facts when she tries to find a cheap iPod on tBay:

$$
\begin{aligned}
& \text { soldBy }(\text { carol, ipod }):-(1,0) . \\
& \text { seller }(t \text { Bay, carol, } u k, 12):-(1,0) . \\
& \text { reputation }(\text { carol, goodSeller, tBay, } 0.9):-\left(\frac{1}{2}, 0\right) . \\
& \text { itemPrice }(\text { ipod }, 80):-(1,0) .
\end{aligned}
$$

Although not signed, Alice considers the information about who is the seller and the price of the item as reliable. However, the reputation is not. Alice knows at least ten ways which sellers can boost their reputation quickly. 
When evaluating the policies, only the assumptions:

recommendation (bob, carol, bid, ipod) :- $(0,0)$

inBlackList(carol) :- $(0,1)$

are used. This is because Alice does not have any relevant information. The other assumptions are not used because Alice has collected the facts and therefore does not need to assume anything.

Let us also explain how the trust (distrust) policies are evaluated. Shinren starts from priority level 3. For the distrust policy at this level, the body is inBlackList(carol) with truth value $(0,1)$ in the interpretation. Therefore distrust(carol,bid, ipod) is evaluated to be $(0,1)$, according to this policy. Because $(0,0) \nprec_{t}(0,1)$, this answer is not admissible and is discarded. The policy with priority 2 does not have an admissible answer either. Given itemPrice $($ ipod, 80$)=(1,0)$, the constraint Price $\leq 20$ is not satisfied because the price is $£ 80$. This constraint is linked to itemPrice(ipod, 80), so its truth value is $\neg(1,0)=(0,1)$. Overall, trust (carol, bid, ipod) is evaluated to $(0,1)$ according to this policy. The answer is also discarded. Because Alice cannot get a recommendation from Bob, the default value is used and the distrust policy at priority level 1 is evaluated to $(0,0)$. The answer is also not admissible. The last policy is evaluated to $\left(\frac{1}{2}, 0\right)$ and therefore is admissible. Because it is a trust policy, we add trust $($ carol, bid, ipod $)=\left(\frac{1}{2}, 0\right)$ and also distrust (carol, bid, ipod $)=\left(0, \frac{1}{2}\right)$ to the model. Alice now knows that although Carol can be trusted, she might still be cheated.

\section{Implementation}

We have implemented a prototype of Shinren. As shown in Figure 3, Shiren consists of five major modules. Among the five modules, the credential module, the recommendation module, the reputation module and the state module are responsible for retrieving and interpreting information from different sources, and the policy interpreter module is responsible for making decisions according to the policies and the information gathered.

The Shinren prototype is implemented in Java 1.5. The policy interpreter evaluates queries in a bottom-up fashion as in many other datalog-based systems. Policies are loaded into the policy interpreter as plain text files. The rules are stratified when they are loaded by analysing the predicate dependency relationships. To answer a query, the interpreter first initialises an interpretation which is an instance of the AssertionSet class. The interpreter queries the other four modules in order to gather facts, i.e. ground instances of the predicates with truth values, which are needed for policy evaluation. The facts are stored in tables related to the predicates in the interpretation. After the interpreter obtains all facts, it constructs the Herbrand universe by collecting all the constants from the query, rules and facts. The interpreter then puts into the initial interpretation assumptions for all the other ground atoms which are in the Herbrand base. It then starts evaluating policies iteratively from the lowest strata. Each rule in the stata is grounded with regard to the Herbrand base and then the interpreter applies the immediate consequence operator to each ground instance. The immediate consequence operator retrieves the truth values for the ground atoms in the rule body from 


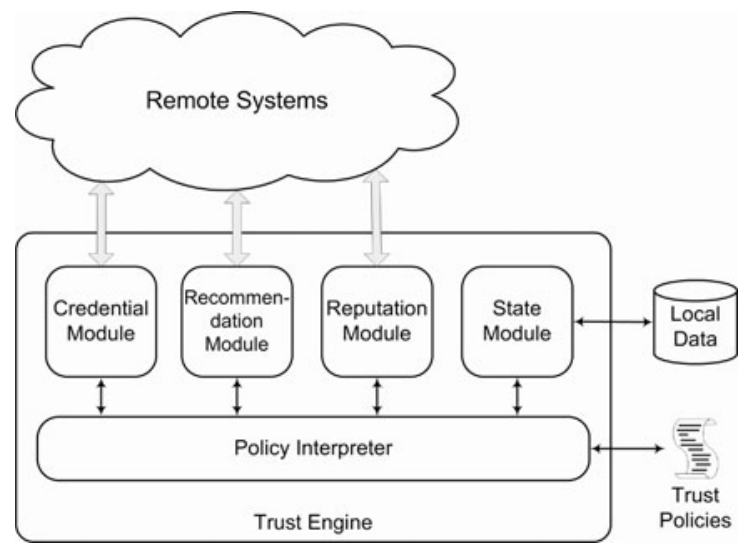

Fig. 3. Shinren Trust Engine

the current interpretation and passes them through the evaluation tree of the rule to obtain the truth value of the ground head atom. The ground atom along with the truth value is a newly generated fact and the table for the head predicate in the interpretation is updated. If an entry with the same ground tuple is already in the table, the truth value of the old entry is ORed with the truth value of the new entry; otherwise the new entry is inserted into the table. Trust (distrust) policies in the same strata are evaluated sequentially by priority level until an admissible answer, i.e. an answer that satisfies the threshold defined for this level, is found. The evaluation of the strata ends when the interpretation does not change anymore. Then the interpreter evaluates the rules in the next strata. The evaluation of the query ends after the interpreter evaluates all the strata containing the rules with the queried atoms as heads.

\section{Conclusion and Future Work}

In this paper, we have presented Shinren, a novel non-monotonic trust management system based on bilattices and the any-world assumption. The syntax of the Shinren policy language is based on Datalog with certain extensions such as negation, constraints and prioritisation. Shinren can utilise unreliable even contradictory information and supports prioritisation which resolves conflicts and provides decision support. We demonstrated the power of Shinren by two comprehensive examples and outlined its implementation. The semantics of Shiren extends the Kripke-Kleene semantics over bilattices and is given in [18].

One aspect that we would like to investigate further is prioritisation. The current prioritisation mechanism in Shinren is at the meta-level. It works but is not convenient in practice because it is external to the bilattice. However, prioritisation can also be viewed as another ordering. We would like to extend our bilattice, so that a third ordering could be integrated into the theory. This would make prioritisation a built-in feature.

The any-world assumption uses the concept of non-uniform assumption. However, its assumptions are static. We are interested in researching dynamic assumptions which 
would mean that changes in knowledge could lead to the change of the assumptions. Dynamic assumptions would enable a trust management system to generate more accurate conclusions according to the context. Previous works in belief revision [19] and dynamic prioritisation [20] are possible stepping stones in this direction.

\section{References}

1. Blaze, M., Feigenbaum, J., Lacy, J.: Decentralized trust management. In: IEEE Symposium on Security and Privacy, pp. 164-173. IEEE Computer Society, Los Alamitos (1996)

2. Mayer, R.C., Davis, J.H., Schoorman, D.F.: An integrative model of organizational trust. The Academy of Management Review 20(3), 709-734 (1995)

3. Blaze, M., Feigenbaum, J., Ioannidis, J., Keromytis, A.D.: The keynote trust-management system, version 2. RFC 2704 (1999)

4. Jim, T.: Sd3: A trust management system with certified evaluation. In: SP 2001: Proceedings of the 2001 IEEE Symposium on Security and Privacy, Washington, DC, USA, pp. 106-115. IEEE Computer Society, Los Alamitos (2001)

5. Li, N., Mitchell, J.C., Winsborough, W.H.: Design of a role-based trust-management framework. In: IEEE Symposium on Security and Privacy, pp. 114-130 (2002)

6. Hess, A., Seamons, K.E.: An access control model for dynamic client-side content. In: SACMAT 2003: Proceedings of the eighth ACM symposium on Access control models and technologies, pp. 207-216. ACM Press, New York (2003)

7. Carbone, M., Nielsen, M., Sassone, V.: A formal model for trust in dynamic networks. In: SEFM, pp. 54-61 (2003)

8. Chu, Y.H., Feigenbaum, J., LaMacchia, B.A., Resnick, P., Strauss, M.: Referee: Trust management for web applications. Computer Networks 29(8-13), 953-964 (1997)

9. Herzberg, A., Mass, Y., Mihaeli, J., Naor, D., Ravid, Y.: Access control meets public key infrastructure, or: Assigning roles to strangers. In: IEEE Symposium on Security and Privacy, pp. 2-14 (2000)

10. Czenko, M., Tran, H., Doumen, J., Etalle, S., Hartel, P., den Hartog, J.: Nonmonotonic trust management for P2P applications. Electronic Notes in Theoretical Computer Science 157(3), 113-130 (2006)

11. Gelder, A.V., Ross, K.A., Schlipf, J.S.: Unfounded sets and well-founded semantics for general logic programs. In: PODS, pp. 221-230. ACM, New York (1988)

12. Marsh, S.P.: Formalising Trust as a Computational Concept. PhD thesis, University of Stirling (1994)

13. Jøsang, A.: A logic for uncertain probabilities. International Journal of Uncertainty, Fuzziness and Knowledge-Based Systems 9(3), 279-212 (2001)

14. Yu, B., Singh, M.P.: Detecting deception in reputation management. In: AAMAS, pp. 73-80. ACM, New York (2003)

15. Ginsberg, M.L.: Multivalued logics: a uniform approach to reasoning in artificial intelligence. Computational Intelligence 4, 265-316 (1988)

16. Loyer, Y., Straccia, U.: Any-world assumptions in logic programming. Theor. Comput. Sci. 342(2-3), 351-381 (2005)

17. Ceri, S., Gottlob, G., Tanca, L.: What you always wanted to know about datalog (and never dared to ask). IEEE Trans. Knowl. Data Eng. 1(1), 146-166 (1989)

18. Dong, C., Dulay, N.: Shinren: Non-monotonic trust management for distributed systems. Technical Report DTR10-5, Department of Computing, Imperial College London (March 2010)

19. Alchourrón, C.E., Gärdenfors, P., Makinson, D.: On the logic of theory change: Partial meet contraction and revision functions. J. Symb. Log. 50(2), 510-530 (1985)

20. Brewka, G.: Reasoning about priorities in default logic. In: AAAI, pp. 940-945 (1994) 


\section{A Example 2: Healthcare in the Community}

Dr Taylor runs a medical clinic in a small town. An unconscious patient is brought to the clinic. From the driver's licence, Dr Taylor learns that the patient is called $\mathrm{Mr}$ Johnson. Mr Johnson is a tourist and stayed in a local hotel before he was brought here. The owner of the hotel, who brought Mr Johnson in, tells Dr Taylor that the patient experienced breathing difficulties during breakfast and then passed out a few minutes later. Dr Taylor examines the patient's trachea and hears the lung sound. He decides to intubate the patient in order to let air pass freely to and from the lungs. The patient's temperature is normal and the results of a blood test show no signs of infection. Blood pressure and heart rate are also normal. Dr Taylor decides to check the patient's medical history in order to see whether the symptoms were caused by drugs or allergies. From his computer, Dr Taylor sends a request to the Smith GP practice, found in documents in Mr Johnson's wallet.

The electronic medical record system of the Smith GP practice uses the Shinren trust management system to control who can access patients' medical histories. The policies which regulate the access to a patient's medical history are shown blow:

$\langle 3\rangle \operatorname{distrust}(X$, read, med_history, $Y):-\neg \operatorname{doctor}($ bma,$X)$.

$\langle 3\rangle \operatorname{trust}(X, r e a d$, med_history,$Y):-$ consent $(Y, X$, read,med_history)

$\langle 3\rangle \operatorname{trust}(X$, read, med_history,$Y):-\operatorname{agent}(Y, Z)$, consent $(Z, X$, read, med_history)

$\langle 2\rangle \operatorname{trust}(X$, read, med_history,$Y):-\operatorname{answer}(X, D O B, A D D R E S S)$, personal_info( $Y, D O B 2, A D D R E S S 2), D O B=D O B 2$, $A D D R E S S=A D D R E S S 2$.

$\langle 1\rangle \operatorname{trust}(X$, read, med_history,$Y):-\operatorname{collocated}(X, Y)$.

Patients' medical histories are sensitive and should only be revealed to doctors who are treating the patients. The distrust policy at level 3 says that $X$ is not allowed to read patient $Y$ 's medical history if $X$ does not have a doctor credential signed by the BMA (British Medical Association). The second trust policy at the same level says $X$ is trusted to read patient $Y$ 's medical history if $Y$ gives his consent. However, in real-life, it is not always possible to get the patient's consent, e.g. in the case that the patient is in coma. Then a third party consent from the patient's agent, usually the next of kin, also has the same effect. In emergency situations where no consent can be obtained, it is necessary to verify that the doctor is indeed treating the patient before letting the doctor access the information without consent. For example, the verification might be done by letting the doctor provide the patient's personal information and comparing it with the data stored, or using a location service to verify that the doctor is co-located with the patient. Accesses without consent are logged and audited.

Dr Taylor provides his doctor credential and also supplies information about $\mathrm{Mr}$ Johnson's birthday and address correctly. The access is granted and logged. Alas the medical history does not provide too much useful information. At the same time, $\mathrm{Mr}$ Johnson's condition becomes worse. He starts to have seizures and EEG (electroencephalogram) shows abnormal brain activities.

Dr Taylor suspects that the problem may be in Mr Johnson's brain. However, he is not a neurologist and needs someone to help in diagnosing the patient. Dr Taylor starts 
looking for help. He searches the NHS database using Shinren with the policies shown below:

$\langle 2\rangle \operatorname{trust}(X$, specialist, neurology) :-consultant(Hos, $X$, neurology), hospital(NHS, Hos), member(aon, X, Level), Level $>=2$.

$\langle 1\rangle \operatorname{trust}(X$, specialist, neurology) :-consultant(Hos, X, neurology), hospital(NHS, Hos), member(aon, X, 1), member (aon, Y, Level), Level > = 2, recommendation $(Y, X$, specialist, neurology).

The first policy says that Dr Taylor will trust $X$ as a specialist in neurology if $X$ has a consultant credential signed by an NHS hospital which states that $X$ is a consultant in neurology. $X$ must also be a member of the Association of Neurologists with level no lower than senior member. The second policy says almost the same except that if the level of $X$ in the Association of Neurologists is not high enough, he needs a recommendation from a senior member or higher.

Dr Taylor finds 20 doctors who fit his requirements. Among them, he selects Dr Ford, a senior member of the Association of Neurologists who works for Victoria Hospital. Dr Ford is also willing to offer assistance. Dr Taylor sets up a video conference with Dr Ford. After hearing the observations and checking the examination results, Dr Ford suggests that the problem could be caused by a clot in the patient's brain. However, a brain tumour also fits the symptoms. The diagnosis can be confirmed by an MRI (Magnetic Resonance Imaging) scan or a brain biopsy. However, the clinic does not have the equipment and the patient's condition is not suitable for transportation. Dr Ford then suggests that in this situation, Dr Taylor should immediately treat the patient with tPA (tissue Plasminogen Activator), a medicine which helps resolving blood clots, because a long delay could cost the patient's life. If the patient's condition gets better, then the diagnosis of a blood clot can be confirmed, otherwise it suggests a brain tumour.

Dr Ford's plan could be quite dangerous. So Dr Taylor wants to hear a second opinion. To ensure the opinion is independent and fair, Dr Taylor adds another policy before he searches for the second specialist. The policy rules out all the specialists working in the same hospital as Dr Ford.

$\langle 2\rangle$ distrust(X, specialist, neurology) :- consultant(victoria, $X$, neurology).

This time Dr Taylor finds Dr Grant, a senior member of the Association of Neurologists who works for the Albert Hospital. Dr Grant confirms that there is no better solution in this situation. Dr Taylor starts to treat the patient with tPA, and watches him closely. 24 hours later, the patient wakes up. After the patient's condition is stabilised, he is transferred to the nearest major hospital for further diagnosis and treatment. 